Results Median days of hospitalization were 3.38 in those $\mathrm{HBoV}$ positive compared to 2.5 in those $\mathrm{HBoV}$ negative. Median hours of $\mathrm{O}_{2}$ requirement in $\mathrm{HBoV}$ positive was 14.23, compared to $\mathrm{HBoV}$ negative that was 5.9. All $\mathrm{HBoV}$ positive patients had an X-ray done, compared with only $58.3 \%$ in those $\mathrm{HBoV}$ negative. The use of antibiotics has been higher in those $\mathrm{HBoV}$ positive $76.92 \%$ compared to those $\mathrm{HBoV}$ negative $58.3 \%$. While in $38.46 \%$ of those $\mathrm{HBoV}$ positive had no other viruses detected, all the $\mathrm{HBoV}$ negative patients had a combination of other viruses detected. Conclusions Patients that tested $\mathrm{HBoV}$ positive needed more resources, compared to those $\mathrm{HBoV}$ negative in all four area assessed. As $\mathrm{HBoV}$ is not completely understood and it is understudied we would need more data to be able to make recommendations regarding testing and treatment for calculating the potential cost for the health system.

\section{P212 CHALLENGES OF LEGALIZATION OF RECREATIONAL MARIJUANA AMONG YOUTH}

Muhammad Akhter Hamid*. Scarborough Health Network, Scarborough, Canada. University of Toronto, Toronto, Canada

10.1136/archdischild-2019-epa.567

Background It is difficult to foresee the challenges that the legalization of recreational cannabis will bring to the youth and future of society. During adolescence, individuals are very susceptible to outside influences, making it easy to be redirected onto a troubling path. Changes in policy for cannabis legalization has the possibility of causing damaging outcomes to the future generation. Currently, ten states in the United States have legalized recreational cannabis use, and Canada has recently become the first country to legalize it. According to a 2013 report by UNICEF, Canadian youth are the highest users of cannabis when compared to students in other developed countries. Yet, despite evidence showing legalization increases regular cannabis use among adolescents, in the United States, there are no significant steps in place to investigate, monitor, and educate these adolescents.

The success in education among young students has also not been heavily considered, with the legalization of marijuana. Some research has even suggested that adolescent cannabis use may be a better indication of lower educational attainment than adolescent alcohol use. There are also no clear guidelines for health-care providers and they struggle to treat young individuals with cannabis use disorders and addiction, as adolescents with cannabis use disorder have shown poor responses to treatment, in terms of abstinence attainment rates. From a long term perspective, structural and functional neuroimaging studies have suggested that adolescent chronic cannabis use may cause morphological alterations in the medial temporal and frontal cortices, and the cerebellum. In addition, since nonconventional methods of marijuana, such as edibles, vape pens, and liquids, access to marijuana becomes easier for adolescents and the ability to manage marijuana use becomes increasingly difficult. Thus, the question remains: Will recreational cannabis legalization hinder the success of future adult members of society?

Objectives The objective of this study is to review literature to evaluate the challenges of legalization of recreational use of Marijuana among youth.
Methods A literature search was conducted through PubMed, MEDLINE, EMBASE, and Cochrane Library. The MeSH headings used were adolescent, youth, cannabis, delta-9-tetra-hydrocannabinol, marijuana, legalization, cognition, and academics. Conclusion The jury's is still out on the outcomes of recreational cannabis legalization among adolescents, however there are already some indications on the potential social and physical harms it may cause. By understanding possible ramifications especially among the youth population, governments, health-care providers, and educational institutions will be able to better equip themselves to these new challenges.

\section{P213 ABSTRACT WITHDRAWN}

\section{P214 ABSTRACT WITHDRAWN}

\section{P215 'HAVE YOU PUT THE FEAR OF GOD INTO ANYONE TODAY?' - AN AUDIT MEASURING SHORT TERM COMPLIANCE WITH ALLERGY CLINIC ADVICE TO INTRODUCE 'SAFE' FOODS}

${ }^{1}$ Molly Cremin*, ${ }^{2}$ Jonathan Hourihane. ${ }^{1} \mathrm{CUH}$, Cork, Italy; ${ }^{2} \mathrm{CUH}$, Cork, Ireland

\subsection{6/archdischild-2019-epa.568}

Aims

- To asses short term compliance with advice to introduce SKIN TEST NEGATIVE foods after clinic.

- To asses barriers to commencing advised consumption.

Methods Allergy-related dietary restrictions are often too broad, and are not based on clinical evidence of allergy or sensitisation. In CUH allergy clinic parents are advised to introduce foods after full allergy focused history, usually negative SPT and occasionally IgE testing. Parents are given a written list of foods to be introduced and are advised that they will be contacted in exactly one week to inquire about (non-) completion of the introductions.

Results 75 parents were contacted 1 week after their clinic visit. 10 parents (13\%) failed to commence food as instructed with reasons such as 'child refusal', 'forgot' and child vomiting immediately post ingestion. 65 (87\%) commenced foods as planned, most were asked to try tree nuts with only 6 parents 13\%) asked to start the egg or milk ladder. 55 of these parents were asked to introduce more than one food (up to 6 foods), 10 of the 55 parents failed to introduce more than one food (18\%) and 45 completed all advised introductions (82\%). Reasons given for incomplete multiple introductions were 'not enough time since clinic' and fear of introducing more than one food would cause a reaction. 47 parents $(72 \%)$ gave the advised foods more than once/regularly in the week, (18) $27 \%$ parents gave the advised foods just once so far, but 13 of these 18 were willing to offer the foods again.

Conclusion $87 \%$ of parent introduced advised food(s) within 1 week, but only $72 \%$ were offering the foods regularly. Reasons for non-compliance were usually not allergy-related and can be worked around, including giving a wider time window for multiple introductions. Introduction of unduly avoided foods is a key part of modern allergy care. 